\title{
Comparación de mediciones antropométricas directa y con sistema de imagen 3D, en adultos jóvenes.
}

\section{Comparison of direct and 3D anthropometric measurements in young adults}

\author{
Sebastián Villanueva-Bonilla ${ }^{1}$, Loreto Saavedra-Layera ${ }^{2 *}$, Cristian Vergara-Núñez ${ }^{3}$.
}

1. Consultorio General Rural Pozo Almonte, Servicio de Salud lquique, Iquique, Chile. 2. CESFAM Padre Vicente Irarrázabal, Servicio de Salud Metropolitano Central, Santiago, Chile. 3. Facultad de Odontología, Universidad de Chile, Santiago, Chile

* Correspondencia autor: Loreto Saavedra-Layera Dirección: Sergio Livingstone 943| Teléfono: $+5666082548$

Trabajo recibido el 19/07/2017. Aprobado para su publicación el 24/12/2017

\section{RESUMEN}

Objetivo: El objetivo de este estudio fue determinar si existe equivalencia entre las medidas faciales obtenidas mediante antropometría facial directa y antropometría facial indirecta utilizando sistema de imagen digital en tres dimensiones en adultos jóvenes. Materiales y métodos: Estudio transversal descriptivo. La muestra consistió en 81 voluntarios (19-29 años). A cada uno se le realizó mediciones de 6 distancias faciales con el método antropométrico directo y 5 distancias faciales con el método fotogramétrico 3D. Se utilizaron los programas Excel 2013 y Stata14®. Se aplicó el test de Shapiro-Wilk, para establecer el tipo de distribución de los datos. Para los que presentaron distribución normal se aplicó t-Test; para aquellos con distribución no normal, se aplicó test de MannWhitney. Se comparó las mediciones obtenidas con ambos métodos.

Resultados: Se compararon las 5 distancias faciales medidas con el método antropométrico directo con las 5 registradas mediante fotogrametría 3D y se determinó que no existe diferencia estadísticamente significativa entre los métodos utilizados. Hubo una medición que no pudo ser registrada con el método fotogramétrico 3D.

Conclusión: La antropometría facial indirecta medida en imágenes 3D, entrega mediciones equivalentes a la antropometría facial directa.

PALABRAS CLAVE

Fotografía dental, Antropometría facial, Estética.

Rev. Clin. Periodoncia Implantol. Rehabil. Oral Vol. 11(1); 16-19, 2018.

\section{ABSTRACT}

Objective: The aim of this study was to determine if there is equivalence between facial measurements obtained through direct facial anthropometry and indirect facial anthropometry using a three dimensional digital imaging system in young adults.

Materials and methods: Descriptive cross-sectional study. The sample consisted of 81 volunteers (19-29 years). Each was measured with 6 facial distances with the direct anthropometric method and 5 facial distances with the 3D photogrammetric method. The programs Excel 2013 and Stata14® were used. The Shapiro-Wilk test was applied to establish the type of distribution of the data. For those with normal distribution, t-Test was applied; for those with non-normal distribution, a Mann-Whitney test was applied. The measurements obtained with both methods were compared.

Results: We compared the 5 facial distances measured with the direct anthropometric method with the 5 recorded by 3D photogrammetry and it was determined that there is no statistically significant difference between the methods used. There was a measurement that could not be recorded with the 3D photogrammetric method.

Conclusion: The indirect facial anthropometry measured in 3D images, provides measurements equivalent to direct facial anthropometry.

KEY WORDS

Dental photography, Facial anthropometry, Esthetics.

Rev. Clin. Periodoncia Implantol. Rehabil. Oral Vol. 11(1); 16-19, 2018.

\section{INTRODUCCIÓN}

La Antropometría facial se refiere al estudio de las medidas y proporciones de la cara de las personas ${ }^{(1,2)}$, la cual es útil en diversas áreas de la odontología para la evaluación de anomalías congénitas, deformidades adquiridas, el diagnóstico, plan de tratamiento, y las comparaciones pre y post-tratamiento o etapas del crecimiento $^{(3,1)}$. La antropometría dispone de dos métodos para obtener información del sujeto en estudio: el método directo que consiste en la medición de puntos en el sujeto por un examinador utilizando instrumentos, el cual requiere habilidades especiales del operador y prolongado tiempo de trabajo, resultando un procedimiento agotador para ambas partes; y el método indirecto en que se encuentran los modelos de yeso y la fotogrametría. Esta última, mediante estandarización de la técnica fotográfica clínica, que consiste en la realización de mediciones en fotografías del sujeto en estudio(4). La antropometría indirecta presenta ventajas frente a la 
antropometría directa ${ }^{(5)}$, tales como: los sujetos en estudio no se mueven, por lo que es más fácil medir. No hay errores en el registro, por ejemplo, por la presión en la piel del instrumento(6). Además, las mediciones pueden realizarse en múltiples ocasiones y la información recopilada puede ser almacenada permanentemente y facilitar el seguimiento del caso a lo largo del tiempo ${ }^{(1)}$.

Una de las principales desventajas de la fotografía es que es una representación bidimensional de una realidad tridimensional, lo que limita su acercamiento a la realidad ${ }^{(7,8)}$.En la actualidad, el rápido desarrollo de los sistemas de imagen en tres dimensiones ha permitido diagnosticar los tejidos blandos faciales en detalle y otorga una nueva posibilidad para su evaluación en la planificación ortodóncica y análisis de los resultados del tratamiento. Estas técnicas de imagen tridimensional son: Cone Beam computed Volumetric Tomography (CBVT), exploración facial superficial láser y estereofotogrametría $3 D^{(9,10)}$.

La estereofotogrametría es un método para obtener una imagen por medio de uno o más pares de estereofotografías tomadas simultáneamente ${ }^{(11)}$. Permite capturar imágenes en menor tiempo, es libre de radiación, identificación exacta de puntos de referencia faciales y proyección inmediata del registro $3 \mathrm{D}$ en una pantalla ${ }^{(12)}$. Esta tecnología presta utilidad en el diagnóstico, la planificación de los tratamientos odontológicos, comunicación con el paciente, comparación pre y posttratamiento, así como el seguimiento del crecimiento y desarrollo del macizo facial ${ }^{(10)}$.

El uso de estereofotogrametría 3D, complementados con un equipo de CBVT que incorpora un dispositivo de escaneo facial 3D, proporcionan en una sesión una fotografía digital en tres dimensiones y volumen CBVT ${ }^{(13)}$. Un ejemplo de dicho equipo, es el Planmeca ProMax $3 D^{\circledR}$ con ProFace ${ }^{\circledR}$ incorporado. La estereofotogrametría 3D permitiría ahorrar tiempo clínico, puesto que, nos faculta para estudiar puntos antropométricos sin que el sujeto en estudio se encuentre presente. Sin embargo, si bien el registro imagenológico del equipo se encuentra estandarizado y las proporciones que entrega son idénticas a las del paciente, existen dudas si la longitud de las medidas que se obtienen a partir de este examen son equivalentes a las reales ${ }^{(13)}$.

Según lo expuesto, la hipótesis de este estudio fue que la antropometría facial indirecta medida en imágenes $3 \mathrm{D}$, entrega mediciones equivalentes a la antropometría facial directa. El propósito fue determinar si existe equivalencia entre las medidas faciales obtenidas mediante antropometría facial directa y antropometría facial indirecta utilizando un sistema de imagen digital en tres dimensiones en adultos jóvenes.

\section{MATERIALES Y MÉTODOS}

Se realizó un estudio transversal descriptivo. Los sujetos fueron seleccionados mediante método no probabilístico por conveniencia. Se contó con 81 voluntarios de ambos sexos, jóvenes adultos, entre los 18 y 29 años de edad (O.M.S, 1986), inscritos como alumno regular de la Facultad de Odontología de la Universidad de Chile. El estudio se realizó siguiendo el acuerdo de Helsinki, en el que los participantes fueron previamente informados y dejaron constancia de ello al firmar un consentimiento informado donde declararon que comprendían la naturaleza del estudio.

Criterios de inclusión: Sujetos ASA I y II. Dentados totales, con referencias oclusales estables.

Criterios de exclusión: Sujetos ASA III o superior. Sujetos sin referencias dentarias, o con rehabilitación protésica removible. Sujetos con síndromes que afecten la estructura maxilofacial. Enfermedades o condiciones invalidantes que dificulten la toma de imágenes y/o medición facial directa.

Medición antropométrica directa: Fue ejecutada por dos investigadores (S.V y M.A) quienes se calibraron realizando seis mediciones a 3 voluntarios, sumando un total de 18 mediciones. Su valor Kappa de Cohen fue de 0,67 ; lo que la literatura describe como un acuerdo moderado. Para la medición se posicionó a cada sujeto de pie, con las manos en un costado. Se le solicitó que fijara la mirada en un punto del horizonte. Se realizó el registro en posición natural de cabeza (PNC), utilizando el protocolo simplificado de Bister ${ }^{(14)}$. Posteriormente se registraron las distancias faciales propuestas en la Tabla 1 y en la Figura 1. En caso de incompetencia labial, se utilizó como referencia el punto Stomion superior en vez de Stomion. Para este procedimiento se utilizó compás de punta seca (Staedtler ${ }^{\circledR}$ Geo Student) y regla milimetrada metálica $\left(\mathrm{COX}{ }^{\circledR} \mathrm{CB}-300\right)$.

Medición antropométrica indirecta con sistema de imagen digital en tres dimensiones: El investigador (S.V), posicionó a los voluntarios en la unidad estereofotográfica 3D (Planmeca ProMax 3D ${ }^{\circledR}$ con ProFace ${ }^{\circledR}$
Tabla 1. Distancias faciales

\begin{tabular}{ll}
\hline \multicolumn{1}{c}{ Plano frontal } & \multicolumn{1}{c}{ Plano lateral } \\
\hline En - En Ancho intercantal & $\begin{array}{l}\text { T-Ex Distancia } \\
\text { tragus-exocanthion }\end{array}$ \\
Al - Al Ancho alar & $\mathrm{N}^{\prime}-$ Sn Altura facial media \\
Sn - Sto Largo labio superior & $\mathrm{Sn}-$ Sto Largo labio superior \\
\hline
\end{tabular}

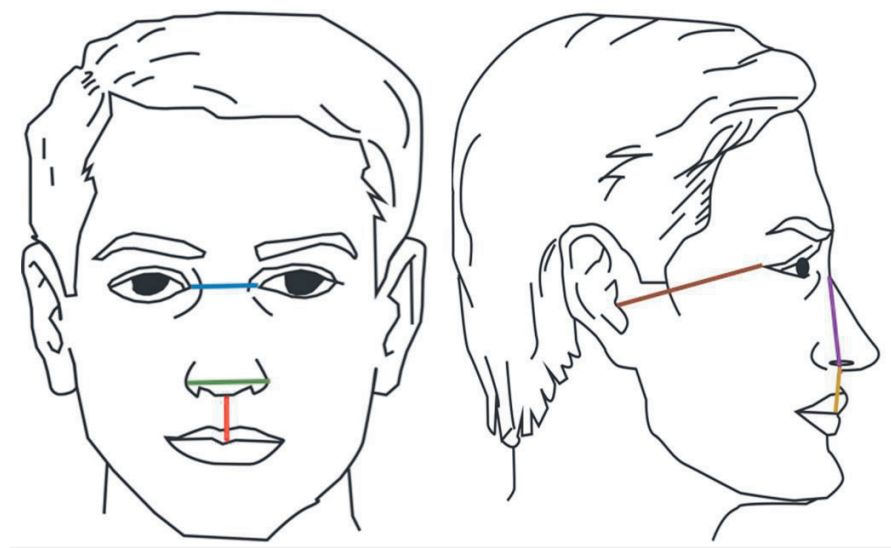

Figura 1. Distancias faciales registradas, presentadas en colores sobre un esquema facial.

incorporado). A cada uno se le indicó poner su cabello detrás de sus orejas, para evitar que este las oculte. Se realizó el registro en posición natural de cabeza (PNC), usando el protocolo simplificado de Bister ${ }^{(14)}$. Adicionalmente, los pacientes debieron permanecer con sus manos en las asas laterales. Se le solicitó mantener su mirada siempre fija, sin pestañar y permanecer inmóvil durante la toma fotográfica.

Las imágenes fueron analizadas por el investigador (S.V) quien se calibró con un experto. Para ello midió 3 fotografías frontales y 3 laterales sumando un total de 18 distancias faciales evaluadas. Su valor Kappa de Cohen fue de 0,87 esto denota un acuerdo fuerte según la literatura. Una semana después, el investigador (S.V) realizó las mismas 18 mediciones para determinar su valor Kappa intraobservador cuyo resultado fue de 0,80 .

Las mediciones fotogramétricas de los voluntarios se realizaron entre 1 a 3 semanas, posterior a las mediciones antropométricas directas. Para ello, se utilizó el visualizador de imágenes de Planmeca Romexis ${ }^{\circledR}$ (Figura 2), luego se utilizó la herramienta de medición de multilínea incorporada en el programa para obtener en milímetros las distancias faciales que se utilizaron en la antropometría directa, exceptuando el plano T - Ex.

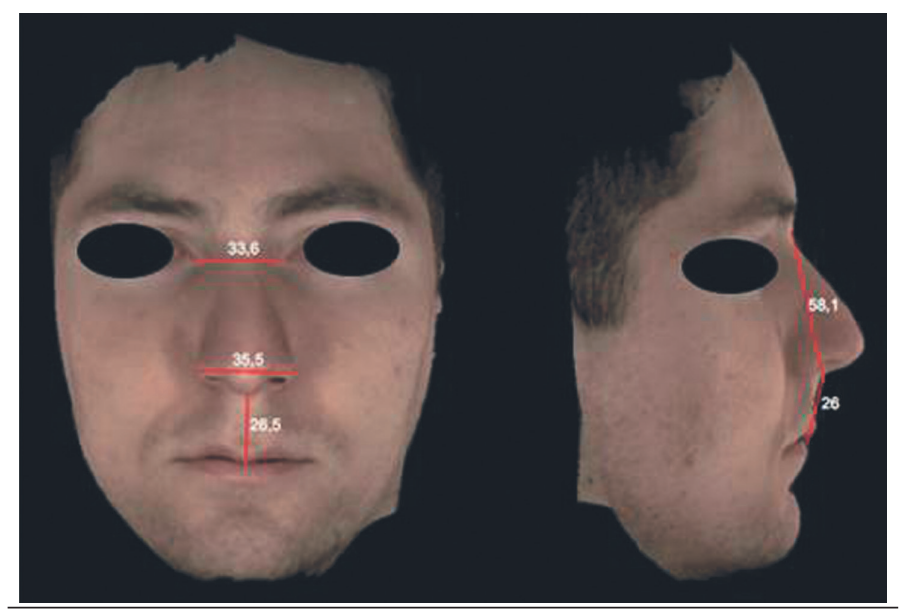

Figura 2. El investigador (S.V) ejemplifica con su rostro la medición fotogramétrica que se llevó a cabo con las imágenes 3D obtenidas de los voluntarios, mediante el uso del visualizador Planmeca Romexis ${ }^{\circledR}$. La unidad de medida fue el milímetro. 


\section{Análisis estadístico}

Los datos se tabularon en una planilla Excel (Microsoft ${ }^{\circledR}$ Excel $\left.^{\circledR} 2010\right)$ y se procesaron estadísticamente con el Software Stata14 S/E. Se aplicó el test de Shapiro-Wilk, para establecer el tipo de distribución de los datos tabulados. Para los que presentaron distribución normal se aplicó t-Test; para aquellos con distribución no normal, se aplicó test de Mann-Whitney.

$\mathrm{N}^{\circ}$ de informe de comité de ética local: 2016/27 del 18 de abril de 2017. Registro de trabajo clínico: PRI-ODO 002/017

\section{RESULTADOS}

El estudio contó con 81 voluntarios, 49 mujeres y 32 hombres. Sus edades estaban entre los 19 y 29 años, el promedio fue 23,7 años y la desviación estándar (Std Dv.) fue 2,18. Se midió con método antropométrico directo a los 81 voluntarios y posteriormente se obtuvo una imagen 3D de cada uno de ellos, las que fueron medidas. En la tabla $\mathrm{n}^{\circ} 2$ se resumen las mediciones obtenidas con ambos métodos.

Al analizar la muestra con test de Shapiro-Wilk se determinó que las mediciones de En-En; Ala-Ala y Sn-Sto presentan distribución normal, a diferencia de N'-Sn (L) y Sn-Sto (L) que poseen una distribución no normal, por lo tanto, se utilizó la pruebas T y de Mann-Whitney, respectivamente, para comparar entre ambos métodos. Esta comparación entregó valores $p$, que son presentados por grupo de mediciones en la tabla $n^{0} 3$. Se puede desprender de esta última tabla que las distancias faciales no presentan diferencia estadísticamente significativa. antropometría facial directa. En acuerdo con la evidencia disponible, los equipos estereofotográficos 3D facilitarían la planificación del tratamiento y la evaluación de cualquier cambio a través del crecimiento o el tratamiento; puesto que, los valores morfológicos de los tejidos blandos faciales se pueden recoger de manera eficiente y medir eficazmente a partir de imágenes capturadas por una cámara 3D no invasiva ${ }^{(10,15)}$. La fotografia 3D elimina la necesidad de registrar por separado los distintos planos proyeccionales, puesto que, estos se registran en una única toma fotográfica y la imagen puede ser manipulada posteriormente ${ }^{(15)}$. Los equipos estereofotográficos $3 \mathrm{D}$ vienen estandarizados de fábrica, no es necesario el ajuste y reposicionamiento del trípode de la cámara para cada paciente y tampoco se necesitaría la iluminación especial que requiere la fotografía $2 \mathrm{D}$, pues posee su propia luz para capturar la imagen sin generar sombras que entorpezcan el registro.

La herramienta de medición incorporada en el programa computacional para visualizar estas imágenes $3 \mathrm{D}$ es de fácil uso, sin embargo, el principal desafío al utilizar imágenes 3D para la evaluación de la morfología facial, es la precisión al ubicar los puntos a estudiar ${ }^{(16)}$. En la presente investigación, la localización de los puntos antropométricos no fue dificultosa en la imagen digital 3D, por tratarse de parámetros anatómicos fácilmente diferenciables. Dentro de las desventajas, la principal es el alto costo del equipo y la gran complejidad de trasladarlo de un lugar a otro ${ }^{(17)}$. Además, el sistema Proface ${ }^{\circledR}$ no logra registrar correctamente las orejas de los pacientes. De las 81 imágenes 3D obtenidas, ninguna registró el punto Tragus. Por este motivo, no fue posible evaluar el plano

Tabla 2. Medidas obtenidas con métodos directo y fotogramétrico 3D, expresadas en milímetros.

\begin{tabular}{|c|c|c|c|c|c|c|}
\hline Distancia & Método & Promedio & Int. Cof. 95\% & Std Dv. & Mínimo & Máximo \\
\hline En - En & Directo & 31,94 & $31,14-32,74$ & 2,43 & 27 & 39 \\
\hline En - En & Indirecto & 32,48 & $31,67-33,29$ & 2,29 & 27,4 & 38,4 \\
\hline Ala - Ala & Directo & 34,74 & $33,87-35,61$ & 3,17 & 27,2 & 42 \\
\hline Ala - Ala & Indirecto & 34,65 & $33,78-35,52$ & 3,50 & 26,5 & 41,2 \\
\hline Sn - Sto & Directo & 21,92 & $21,37-22,47$ & 2,64 & 16 & 29 \\
\hline Sn - Sto & Indirecto & 22,58 & $22,02-23,14$ & 2,56 & 15,7 & 28,4 \\
\hline$N^{\prime}-S n(L)$ & Directo & 52,02 & $50,72-53,32$ & 4,11 & 37 & 61 \\
\hline$N^{\prime}-S n(L)$ & Indirecto & 52,28 & $50,97-53,59$ & 3,27 & 45,4 & 58,8 \\
\hline Sn - Sto $(L)$ & Directo & 22,49 & $21,93-23,05$ & 2,75 & 17 & 31 \\
\hline Sn - Sto (L) & Indirecto & 21,59 & $21,05-22,13$ & 2,70 & 15,6 & 31,4 \\
\hline
\end{tabular}

(L) señala que la medida se registró desde el plano lateral. Unidad de medida: milímetro

Tabla 3. Comparación entre métodos directo e indirecto

\begin{tabular}{c|c}
\hline Distancia facial & Valor $\mathbf{p}$ \\
\hline En - En & 0,08 \\
Ala - Ala & 0,43 \\
Sn - Sto & 0,06 \\
N' - Sn (L) & 0,69 \\
Sn - Sto (L) & 0,05
\end{tabular}

Poder estadístico de 80\%. Nivel de significación de 0,05.

\section{DISCUSIÓN}

La antropometría directa es un modo clásico y confiable de medición, que no requiere elementos sofisticados para su desarrollo; mientras que, la fotogrametría mediante sistema de imagen 3D es un método moderno de medición, que entrega una reproducción digital fidedigna del sujeto en estudio.

Al realizar el análisis estadístico de los resultados obtenidos en el presente estudio, podemos afirmar que la antropometría facial indirecta medida en imágenes $3 \mathrm{D}$, entrega mediciones equivalentes a la
T - Ex en las fotografías 3D. Por otra parte, la utilización del soporte para el mentón en el posicionamiento del paciente, oculta la zona anatómica y podría alterar el registro de los tejidos blandos próximos a este. El empleo del soporte frontal y del soporte para el mentón que posee el equipo no permiten el registro en PNC, puesto que, al brindar puntos de apoyo, la postura no refleja un equilibrio fisiológico natural de la cabeza ${ }^{(13)}$. Es importante generar un protocolo para la obtención de fotografías 3D, que aclare el uso de los elementos de soporte para el paciente. De este modo, el operador del equipo esterofotográfico 3D podrá tener una guía clara, que ayude a obtener resultados óptimos, así como, facilitar la toma de decisiones para un trabajo más eficiente.

A partir de los resultados obtenidos en este estudio, se concluyó que la antropometría facial indirecta medida en imágenes $3 \mathrm{D}$, entrega mediciones equivalentes a la antropometría facial directa. Esto demuestra que la imagen 3D proporciona un medio por el cual se puede evaluar con precisión la morfología facial. La estereofotogrametría 3D es una herramienta sencilla, rápida y efectiva para realizar estudios de antropometría facial. Este método abre nuevas posibilidades al permitir la sobreposición de imágenes en tres dimensiones del rostro de los pacientes y de los tejidos duros subyacentes.

Se sugieren futuros estudios que comparen las mediciones de distancias faciales, obtenidas con los métodos antropométricos indirectos mediante fotografía digital 2D y la estereofotografía 3D, para establecer una relación certera entre ambos. 


\section{Bibliografía}

1. Han K, Kwon HJ, Choi TH, Kim JH, Son D. Comparison of anthropometry with photogrammetry based on a standardized clinical photographic technique using a cephalostat and chair. J Craniomaxillofac Surg. 2010; 38(2):96-107.

2. Nechala P, Mahoney J, Farkas LG. Digital two-dimensional photogrammetry: a comparison of three techniques of obtaining digital photographs. Plast Reconstr Surg. 1999; 103(7):1819-25.

3. Sheridan P. Practical aspects of clinical photography: part 1--principles, equipment and technique. ANZ J Surg. 2013; 83(3):188-91.

4. Farkas, L. G. Accuracy of anthropometric measurements: past, present, and future. Cleft Palate Craniofac J. 1996; 33: 10-8, 19-22.

5. DiSaia, J. P., Ptak, J. J. \& Achauer, B. M. Digital photography for the plastic surgeon. Plast Reconstr Surg. 1998; 102: 569-73.

6. Farkas, L. G., Bryson, W. \& Klotz, J. Is photogrammetry of the face reliable? Plast Reconstr Surg. 1980; 66: 346-55

7. Ahmad I. Digital dental photography. Part 1: an overview. Br Dent J. 2009; 206(8):403-7.

8. Portugal M, Guzzo S, Rodríguez A. Los materiales fotográficos: su organización y tratamiento en la biblioteca. Inf Cult Soc. 2003; (8): 85-105.

9. Baysal A, Sahan A, Ozturk M, Uysal T. Reproducibility and reliability of three-dimensional soft tissue landmark identification using three-dimensional stereophotogrammetry. Angle Orthod. 2016; 86(6): 1004-1009.
10. Othman S. A., Ahmad R, Mericant A. F., Jamaludin M. Reproducibility of facial soft tissue landmarks on facial images captured on a 3D camera. Aust Orthod J. 2013; 29: 58-65

11. Gwilliam J, Cunningham S, Hutton T. Reproducibility of soft tissue landmarks on three-dimensional facial scans. Eur J Orthod 28. 2006; 408-415.

12. Ayoub A, Wray D, Moos K, Siebert P, Jin J, Niblett T, Urquhart C, Mowforth

$\mathrm{R}$. Three-dimensional modeling for modern diagnosis and planning in maxillofacial surgery. Int J Adult Orthodon Orthognath Surg. 1996; 11(3).

13. Moraga, V. Comparación de proporciones faciales obtenidas mediante sistema fotográfico digital 3D y antropometría directa [tesis de pregrado], Santiago, Universidad de Chile, 2013.

14. Bister, D., Edler, R. J., Tom, B. D. \& Prevost, A. T. Natural head postureconsiderations of reproducibility. Eur J Orthod. 2002; 24: 457-70.

15. Ghoddousi H, Edler R, Haers P, Wertheim D, Greenhill D. Comparison of three methods of facial measurement. Int. J. Oral Maxillofac. Surg. 2007; 36: 250-258.

16. Khambay B, Nairn N, Bell A, Miller J, Bowman A, Ayoub A. Validation and reproducibility of a high-resolution three-dimensional facial imaging system. $\mathrm{Br} J$ Oral Maxillofac Surg. 2008;46(1): 27-32.

17. Ozsoy U, Demirel BM, Yildirim FB, Tosun O, Sarikcioglu L. Method selection in craniofacial measurements: advantages and disadvantages of 3D digitization method. J Craniomaxillofac Surg. 2009; 37(5): 285 - 290. 